\title{
Editorial
}

\section{Low vision: Rescue, regeneration, restoration and rehabilitation}

\author{
Andrea Antal ${ }^{\mathrm{a}}$ and Bernhard Sabel ${ }^{\mathrm{b}}$ \\ ${ }^{\mathrm{a}}$ University Göttingen, Göttingen, Germany \\ ${ }^{\mathrm{b}}$ Institute of Medical Psychology, Otto-v.-Guericke University of Magdeburg, Magdeburg, Germany
}

\section{Dear Colleagues,}

The aim of this special issue is to present topics related to vision loss after damage to the retina, optic nerve or brain. Most of the authors were invited speakers at the conference "Low Vision and the Brain", held in Berlin in 2017 and 2018 (mycharwww.4r-vision.com). These visual conferences were unique as they addressed the eye-brain interaction in an interdisciplinary manner, touching the fields not only from the view of ophthalmology and neurology but also of neuropsychology, rehabilitation medicine, engineering and pharmacology. During the talks and related discussions we realised that the topic of low vision is gaining scientific momentum. However, despite remarkable progress in the last and recent years, this subject deserves more attention.

Neurodegeneration of the visual system with subsequent vision loss has very particular challenges in clinical management as so many patients are left with permanent visual problems because there are few effective treatments. The exponential progress in science and technology gives rise towards an optimistic future of prevention, compensation and even restoration of low vision. In this special issue he contributing authors summarize recent developments, highlighting basic science, diagnostic and treatment approaches of this field. These advances impact different visual disorders including typical eye-related diseases such as glaucoma and optic neuropathy, and also more brain-related disorder, such as stroke or brain trauma. Indeed, besides the retina the brain's visual system is also a potential target to offer new research and therapy opportunities, which might greatly influence patient care in the future.

The first part of this special issue presents seven articles, covering the above-mentioned fields from theoretical, experimental and clinical viewpoints. Related to basic research, Benowitz et al summarize the most important results related to optic nerve regeneration; Huang et al. review current knowledge about the PTEN/AKT/mTOR pathway and discuss future directions of translational regenerative research for neural injury and neurodegenerative diseases. From the clinical point of you, the review of Vaucher et al. targets the translational aspects of visual restoration, examining whether potentiating the central cholinergic system could help visual perception and restoration in rats and patients; $\mathbf{L i}$ et al. discuss the possible mechanisms underlying exercise-elicited protection on retina, presenting upto-date findings from both human and animal studies. Pitchaimuthu et al. address the effect of congenital visual deprivation on color vision using a panel based color arrangement test; Werth recaps the causes of dyslexia and gives a summary with regard to identifying effective compensatory therapy in this disorder and Auvray in her paper introduces sensory substitution devices.

We believe that the publications of a dedicated special issue is timely and will significantly advance the field, providing a source of inspirations as the basis of progress. 


\section{References}

Auvray, M. (2019). Multisensory and spatial processes in sensory substitution. Restorative Neurology and Neuroscience, 37(6), 609-619. DOI 10.3233/RNN-190950

Huang, H., Kaur, S., \& Hu, Y. (2019). Lab review: Molecular dissection of the signal transduction pathways associated with PTEN deletion-induced optic nerve regeneration. Restorative Neurology and Neuroscience, 37(6), 545-552. DOI 10.3233/RNN-190949

Li, H.-Y., Rong, S.-S., Hong, X., Guo, R., Yang, F.-Z., Liang, Y.Y., Li, A., \& So, K.-F. (2019). Exercise and retinal health. Restorative Neurology and Neuroscience, 37(6), 571-581. DOI 10.3233/RNN-190945

Pitchaimuthu, K., Sourav, S., Bottari, D., Banerjee, S., Shareef, I., Kekunnaya, R., \& Röder, B. (2019). Color vision in sight recovery individuals. Restorative Neurology and Neuroscience, 37(6), 583-590. DOI 10.3233/RNN-190928

Vaucher, E., Laliberté, G., Higgins, M.-C., Maheux, M., Jolicoeur, P., \& Chamoun, M. (2019). Cholinergic potentiation of visual perception and vision restoration in rodents and humans. Restorative Neurology and Neuroscience, 37(6), 553-569. DOI 10.3233/RNN-190947

Werth, R. (2019). What causes dyslexia? Identifying the causes and effective compensatory therapy. Restorative Neurology and Neuroscience, 37(6), 591-608. DOI 10.3233/RNN-190939

Yin, Y., De Lima, S., Gilbert, H.-Y., Hanovice, N.J., Peterson, S.L., Sand, R.M., Sergeeva, E.G., Wong, K.A., Xie, L., \& Benowitz, L.I. (2019). Optic nerve regeneration: A long view. Restorative Neurology and Neuroscience, 37(6), 525-544. DOI 10.3233/RNN-190960 